\title{
Exercício Físico, Atividade Física e os Benefícios Sobre a Memória de Idosos
}

\author{
Physical Exercise, Physical Activity and the Benefits of the Memory of Elderlys \\ Ejercicio Físico, Actividad Física y los Beneficios sobre la Memoria de Anciano \\ Helder Chiari \\ Marco Túlio de Mello \\ Patricia Rezeak \\ Hanna Karen M. Antunes \\ Universidade Federal de São Paulo, UNIFESP
}

\begin{abstract}
Resumo
Tem sido proposto que a atividade física e exercícios físicos seriam um meio eficiente e de baixo custo para a prevenção dos déficits cognitivos vistos no processo de envelhecimento. Nesse contexto, a atual revisão objetiva avaliar as evidências dos benefícios da atividade física e dos exercícios físicos sobre a memória de idosos. Foram acessados artigos que analisaram os efeitos de um estilo de vida ativo sobre a memória, bem como a resposta aguda e crônica da memória aos exercícios aeróbios e resistidos, as comparações dos efeitos destas modalidades de exercícios sobre a cognição e as diferentes combinações dos componentes do exercício sobre a memória. Concluise que existem fortes evidências de que a atividade física e os exercícios promovem benefícios à memória, porém ainda existem lacunas no conhecimento.
\end{abstract}

Palavras chave: atividade física; exercício físico; memória; cognição; envelhecimento.

\begin{abstract}
Has been proposed that physical activity and physical exercise can promove an effective means with low cost for the prevention of cognitive deficits in the ageing process. In this context, the current review aim to assess the evidences of benefits from physical activity and exercise to aging memory. Were accessed studies that examine the effects of an active lifestyle on memory as well as the acute and chronic, aerobic and resistance exercise on memory's answer, the effects' comparisons of these exercises modalities on cognition and the different combinations of exercise components on the memory. Concluded that there is compelling evidence that physical activity and exercises promoting benefits to memory but there are many gaps in knowledge.
\end{abstract}

Key Words: physical activity, physical exercise, memory, cognition, ageing.

\section{Resumen}

Ha sido propuesto que la actividad física y los ejercicios físicos serían eficientes y de bajo costo en al prevención de los déficits cognoscitivos observados en el proceso de envejecimiento. En este contexto, la presente revisión tiene como objetivo evaluar pruebas de las ventajas de la actividad física y de los ejercicios físicos en la memoria de ancianos. Han sido revisados artículos que han analizado los efectos de un estilo de vida activo en la memoria, así como la respuesta aguda y crónica de la memoria a los ejercicios aeróbicos y de resistencia, las comparaciones de los efectos de estas clases de ejercicios en la cognición y las diferentes combinaciones de los ejercicios sobre la memoria. Se ha concluido que ha fuertes evidencias de que la actividad física y los ejercicios promueven beneficios a la memoria, sin embargo todavía hay vacíos en el conocimiento.

Palabras clave: actividad física; ejercicio físico; memoria; cognición; envejeciéndo.

\section{Introdução}

Mesmo na ausência de patologias predisponentes, $\mathrm{o}$ declínio das funções cognitivas são uma conseqüência normal no processo de envelhecimento. Embora essas alterações cognitivas possam variar entre indivíduos quanto a sua intensidade e domínios cognitivos mais comprometidos, elas podem ser detectadas mais objetivamente a partir da $5^{\text {a }}$ década de vida, quando alterações cerebrais nas células nervosas, vasculatura, morfologia e nos neurotransmissores podem ser

1 Endereço para Correspondência: R. Marselhesa, 535 Vila Clementino São Paulo- SP - 04020-060

e-mail: hanna.karen@unifesp.br observadas (Peters, 2006).

Individualmente, o declínio cognitivo tem um impacto relevante sobre a qualidade de vida e está associado com um risco mais elevado para as limitações funcionais e dependência de terceiros. Coletivamente, pode estar envolvido com a ocorrência de quadros demenciais e o aumento dos índices epidemiológicos desta patologia que sabidamente onera os sistemas de saúde. Desta forma, acredita-se que intervenções eficazes para impedir ou retardar a taxa do declínio cognitivo beneficiariam extremamente o indivíduo e a sociedade (Van Uffelen, Chin A Paw, Hopman-Rock \& Van Mechelen, 2008).

Dentre as queixas subjetivas de declínio 
cognitivo mais comum em idosos encontram-se o comprometimento da memória (Craik et al., 2007). A memória refere-se ao processo complexo pelo qual o indivíduo codifica, armazena e recupera informações e conhecimentos. A codificação está relacionada com o processamento da informação enquanto que a consolidação envolve o processo de fortalecimento das representações já armazenadas. Mas para a memória ser útil, o indivíduo deve ser capaz de recuperar a informação quando esta for necessária (Banich, 2004).

A memória não é um sistema unitário, mas consiste de uma variedade de formas diferentes, cada uma mediada por diferentes componentes que são por sua vez mediados por diferentes mecanismos neurais (Moscovitch, 2004). Uma forma de classificar a memória é pelo seu tempo de duração. Assim a memória imediata, ou de curto prazo, é caracterizada pela capacidade de usar imediatamente a informação aferente apresentada tendendo a ser perdida depois de decorridos alguns segundos do estímulo; a memória recente, cujas informações estão armazenas há algumas horas ou dias; e a remota, aquela que se consolidou há semanas, meses ou anos (Rosat, Chaves, Ribeiro \& Izquierdo, 1990). Este último tipo de memória pode ser armazenado por tempo indeterminado e é regida pela freqüência de repetição do evento e pela capacidade de armazenamento e retenção de informações inerente a cada individuo (Gleitman, 2002).

Ainda não há consenso quanto aos mecanismos de consolidação da memória. No entanto, já está estabelecido que esse processo é modulado pela ação de fibras excitatórias glutamatérgicas e colinérgicas e de fibras inibitórias gabaérgicas que agem sobre a amígdala, o hipocampo, o septo medial e o córtex entorrinal, áreas que estão envolvidas na consolidação, armazenamento e evocação da memória (Izquierdo \& Medina, 1993). Sabe-se que o sono tem um papel fundamental neste processo, fato que se apóia justamente no fenômeno de que as experiências vividas não se fixam instantaneamente, mas sofrem influências neuromodulatórias por várias horas após a aquisição e exigem síntese protéica para se tornarem memórias de longo prazo (Guzowski et al., 2000)

Notadamente, alguns hormônios que desempenham um importante papel na regulação da memória também promovem função adaptadora do organismo às condições estressantes provocadas pelo exercício físico. Dentre estes hormônios encontramse àqueles que se relacionam ao sistema nervoso simpático e às glândulas adrenais, sugerindo que o exercício possa influenciar esse processo (Mazzeo, 1991), porém, ainda não há consenso quanto aos mecanismos envolvidos nesse evento. Entre as hipóteses encontram-se (i) o aumento dos níveis plasmáticos de cálcio que através de um sistema calmodulina-dependente regularia várias funções do cérebro (Sutoo \& Akiyama, 2003) e (ii) o efeito do exercício no aumento do Brain-derived neurotrophic factor (BDNF), um fator neurotrófico que participa da transmissão, modulação e plasticidade neuronal (Ferris, Willians \& Shen, 2007). Esses fenômenos vêm rendendo à atividade física (ATF) e aos exercícios físicos um papel na prevenção e tratamento dos déficits cognitivos progressivos observados no processo de envelhecimento (Peters, 2006).

Essa revisão objetiva analisar a efetividade dos benefícios da ATF e exercícios físicos sobre a memória idosos e analisar se já é possível estabelecer diretrizes para a elaboração de programas de tratamento/ treinamento com vistas à memória.

A pesquisa foi feita nas bases de dados Pubmed, Science Direct, Cochrane, Ebsco Host, Ingenta Connect e Scopus. A busca da literatura foi conduzida utilizando a seguinte combinação de palavras-chave: cognição, memória, exercício ou atividade física. Foram selecionados apenas artigos na língua inglesa publicados entre 1970 e maio de 2009.

\section{Estudos prospectivos}

Estilos de vida mais fisicamente ativos parecem trazer benefícios à memória de idosos saudáveis ou portadores de patologias que acometem o sistema cognitivo (Wiles, Haase, Gallacher, Lawlor \& Lewis, 2007). Estudos prospectivos demonstram que esses benefícios podem estar relacionados aos efeitos da atividade física sobre o sistema cardiovascular (Barnes, Yaffe, Satariano \& Tager, 2003) ou mesmo com fatores genéticos e ambientais (Johnson, Deary, McGue \& Christensen, 2009).

Entre os anos de 1980 e 2001, Verghese et al. (2003), acompanharam a freqüência em atividades físicas e de lazer em idosos com idade igual ou superior a 75 anos e demonstraram que àqueles com maior participação em atividades recreacionais e físicas apresentavam menores probabilidades de desenvolverem processos demenciais, fato creditado às atividades recreacionais, uma vez, que apesar dos benefícios da ATF na promoção da saúde física serem evidentes, sua proteção contra a demência ainda era incerta.

Estudos conduzidos com metodologia similar observaram que um estilo de vida ativo diminui os efeitos deletérios do declínio cognitivo. Larson et al. (2006) acompanharam a freqüência de ATF de 1.740 voluntários com 65 anos ou mais durante nove anos. Apesar deste estudo ter avaliado apenas a freqüência da ATF e não ter medidas adequadas da intensidade das mesmas, os resultados revelam que pessoas que se exercitavam três vezes por semana ou mais, comparadas com aquelas que se exercitavam menos de três vezes por semana, apresentavam menores incidência de demência. Resultados semelhantes já haviam sido encontrados antes por Weuve et al. (2004), quando 18.766 enfermeiras foram acompanhadas entre os anos 1995 e 2001. O objetivo do acompanhamento 
era relacionar as ATF, como caminhadas e atividades de baixa intensidade (alongamentos e yoga, por exemplo), com o funcionamento cognitivo. Além dos questionários para avaliação cognitiva e de ATF os pesquisadores estimavam ainda o gasto calórico despendido em atividades de vida diária. Os resultados demonstraram uma relação inversa entre os níveis de ATF e declínio cognitivo. Especificamente, as voluntárias com altos níveis de ATF pareciam serem três anos de idade mais jovem e tinham $20 \%$ menos chances de desenvolver déficits cognitivos.

Estudos ainda sugerem que os benefícios da ATF sobre a memória sejam mais evidentes após a $5^{\text {a }}$ década de vida. Pesquisadores avaliaram a freqüência de atividades de lazer e ATF de 1.919 sujeitos a partir dos 36 anos de idade que foram submetidos a testes cognitivos aos 43 e 53 anos. Aos 43 anos observouse que àqueles com piores desempenhos cognitivos eram os voluntários que menos praticavam atividades de lazer e ATF. Além disso, embora os participantes que praticavam atividades de lazer tivessem melhor desempenho do que aqueles que praticavam ATF foi a associação entre ambas as atividades que demonstraram os melhores resultados. No entanto, quando os voluntários foram avaliados aos 53 anos de idade, aqueles que praticavam exercícios, independente das atividades de lazer, apresentavam as menores taxas de declínio da memória (Richards, Hardy \& Wadsworth, 2003).

\section{Estudos transversais}

As diversas variáveis nos estudos transversais demonstram que os benefícios da ATF sobre a memória podem estar relacionados com a manutenção da saúde cardiovascular, estilo de vida (Gallucci et al., 2009), ou com a funcionalidade. Kimura et al. (2009) mediu esse último parâmetro em 147 idosos com idade de 60 anos ou mais e encontrou uma correlação significante com a memória. Entendeu-se por funcionalidade neste estudo, o tempo necessário para os voluntários se levantarem de uma cadeira e caminharem naturalmente por três metros, retornarem e sentarem novamente na cadeira. Os pesquisadores concluíram que a manutenção da mobilidade física pode diminuir a velocidade de declínio da memória no processo de envelhecimento.

Metodologia semelhante foi utilizada por Nieto, Albert, Morrow e Saxton (2008), que observaram que os voluntários com os menores índices de memória e funções executivas apresentavam valores até quatro vezes menores de função dos membros inferiores.

Nos estudos de Lambourne (2006) e de Newson e Kemps (2006), a freqüência de ATF (corrida, natação e jogos) e de atividades de lazer (prática de leitura de livros, caça-palavras e estudo) foram avaliadas por meio de questionários em voluntários de ambos os gêneros, com idades entre 18 e 92 anos. Os resultados foram comparados aos testes cognitivos e demonstraram que os melhores desempenhos nas tarefas cognitivas estavam relacionados aos maiores níveis de ATF. No estudo de Newson e Kemps (2006) foram aplicados testes cognitivos de complexidade diferentes e os pesquisadores observaram que o bom desempenho nas tarefas mais complexas estavam fortemente associadas às maiores freqüências de ATF e de atividades cognitivas.

\section{Efeitos do exercício aeróbio sobre a memória}

Tem sido proposto que mesmo uma sessão de exercício aeróbio é capaz de alterar o funcionamento da memória. Netz, Tomer, Axelrad, Argov e Inbar (2007) observaram melhoras cognitivas em idosos cinco minutos após a realização de exercícios aeróbios em intensidade de 60 ou $70 \%$ da Freqüência Cardíaca Reserva (FCR) durante 35 minutos. Recentemente, Kamijo, Nishihira, Higashiura e Kuroiwa (2009) avaliaram a memória dois minutos depois de idosos e jovens realizaram 20 minutos de exercícios a 30 e $50 \%$ do VO2máx em dias separados. Como a magnitude dos benefícios pós exercício em relação ao pré exercício foram similares entre jovens e idosos, os autores propuseram que o efeito agudo do exercício sobre a cognição pode ser observado em todas as faixas etárias.

Por sua vez, o treinamento com exercícios aeróbios tem demonstrado algumas controvérsias. No estudo de Kara, Pinar, Uğur e Oğuz (2005), 45 mulheres saudáveis com idades entre 60 e 80 anos que foram submetidas ao treinamento aeróbio com exercícios calistênicos, três vezes por semana durante quatro meses, apresentaram uma correlação significante entre a melhora da capacidade aeróbia e da cognição. Fabre, Chamari, Mucci, Massé-Biron e Préfaut (2002) compararam quatro grupos de idosos: exercícios aeróbios, treinamento mental, combinado (treinamento mental e exercícios aeróbios) e um grupo controle. O grupo de exercícios aeróbios e o grupo combinado realizaram seus treinamentos com intensidade equivalente ao limiar ventilatório um por uma hora semanal durante dois meses. No mesmo período, o grupo de treinamento mental e o combinado foram submetidos a 90 minutos semanais de uma terapia que visava estimular a memória a partir de estímulos auditivos e visuais. O grupo controle realizou várias outras atividades como pintar ou cantar. Ao final do estudo, somente o grupo controle não apresentou melhoras na memória, sendo que os melhores resultados foram obtidos pelo grupo de atividades combinadas (exercício e treino mental). Porém, quando comparada à evolução da memória entre os grupos de atividades aeróbias e mentais, os melhores resultados foram observados no grupo que realizou exercícios.

A controvérsia fica por conta do estudo de Madden, Allen, Blumenthal e Emery (1989), que apesar de utilizarem um programa de treinamento similar ao de 
Tabela 1

Efeitos do treinamento com exercício aeróbio sobre a memória

\begin{tabular}{|c|c|c|c|}
\hline Autor & Voluntários & Prog. treino & Resultados \\
\hline Kara et al, 2005 & $\begin{array}{l}\text { Mulheres } \\
60-80 \text { anos }\end{array}$ & $\begin{array}{l}4 \text { meses, 3Xsem } \\
20-30 \text { min } \\
60-70 \% \text { FCmáx }\end{array}$ & melhora aeróbia $=$ melhora cognição \\
\hline Fabre et al, 2002 & $\begin{array}{l}\text { Ambos } \\
60-70 \text { anos }\end{array}$ & 2 meses, 1h/semana, LV1 & $\begin{array}{l}\text { melhora condição aeróbia }=\text { melhora } \\
\text { memória }\end{array}$ \\
\hline $\begin{array}{l}\text { Madden et al, } \\
1989\end{array}$ & $\begin{array}{l}\text { Ambos } \\
65-83 \text { anos }\end{array}$ & $\begin{array}{l}4 \text { meses } \\
3 \text { Xsem, } 45 \mathrm{~min} \\
\text { (30'esteira e } 15 \text { ' jogos } \\
\text { lúdicos), } 70 \% \text { FCmáx }\end{array}$ & $\begin{array}{l}\text { melhora VO2 } \\
\text { porém sem melhoras cognitivas }\end{array}$ \\
\hline
\end{tabular}

Fabre et al. (2002) e observarem melhoras nos níveis de VO2, não observaram melhoras cognitivas (Tabela $1)$.

\section{Efeitos do exercício resistido sobre a memória}

Existem poucos trabalhos que observaram os efeitos agudos do exercício resistido sobre a cognição e, em sua maioria, os autores não se limitaram a avaliar esses efeitos em idosos. Chang e Etnier (2009) estudaram sujeitos com idades entre 35 e 60 anos, antes e depois de serem submetidos a duas séries de 10 repetições em seis exercícios resistidos com intensidade correspondente a $75 \%$ de $1 \mathrm{RM}$ (Repetição máxima). Após o exercício, o desempenho dos voluntários nos testes que avaliam habilidades executivas como a memória foram superiores, independente da complexidade da tarefa realizada, porém, não foi observada melhora de desempenho nos testes para flexibilidade cognitiva, sugerindo indícios de que pode haver um efeito específico do exercício agudo sobre a cognição.

No estudo de Cassilhas et al. (2007), idosos (65 a 70 anos) treinados com exercícios resistidos feitos a 50 ou $80 \%$ de 1 RM apresentaram melhoras cognitivas após 24 semanas do protocolo. Neste estudo, um grupo de idosos realizaram uma hora de exercícios, três vezes por semana, e um grupo controle realizou o mesmo protocolo do grupo experimental, uma vez por semana, porém, os exercícios eram feitos sem carga. Essa estratégia com o grupo controle foi adotada para evitar a possível interferência da aprendizagem neuromotora e da interação social nos resultados.

Em outro ensaio clínico, os exercícios resistidos foram realizados uma vez por semana em oito aparelhos durante oito semanas. Após o protocolo, os idosos de ambos os gêneros apresentaram aumento na força muscular e uma melhora na memória, mantida por até um ano após o início do estudo, quando comparado com o grupo controle, não submetido ao exercício resistido (Perrig-Chiello, Perrig, Ehrsam, Staehelin \& Krings, 1998).

\section{Comparações da resposta do exercício resistido e do exercício aeróbio sobre a memória}

A comparação entre os efeitos agudos do exercício resistido e aeróbio sobre a memória foi visto em jovens com idade média de 20 anos, de ambos os gêneros, submetidos em dias diferentes, mas no mesmo horário, a 30 minutos de exercício resistido, 30 minutos de exercício aeróbio e 30 minutos de repouso. A cada dia os participantes realizavam teste de memória antes, imediatamente depois e trinta minutos após o experimento. Os exercícios aeróbios foram realizados a uma intensidade de 60 a $70 \%$ do $\mathrm{VO}_{2}$ máx, enquanto a sessão de exercícios resistidos consistia de oito a 12 repetições a $80 \%$ de 1 RM para cada um dos sete maiores grupos musculares. Os resultados demonstraram que imediatamente e 30 minutos após o exercício, houve uma melhora nos testes de memória apenas no dia em que os voluntários realizaram exercícios aeróbios, porém a magnitude dos resultados dependia da exigência da tarefa realizada, suportando a hipótese de que a resposta aguda da cognição ao exercício depende da natureza da tarefa cognitiva exigida ao voluntário (Pontifex, Hillman, Fernhall, Thompson e Valentini, 2009).

A comparação entre os efeitos do treinamento com exercícios resistidos e aeróbios sobre a memória também foi realizada em adultos e idosos. Blumenthal e Madden (1988) observaram melhoras em adultos após 12 semanas de treinamento aeróbio ou anaeróbio. $\mathrm{O}$ treinamento aeróbio era realizado três vezes por semana, com duração de 30 a 45 minutos, em uma intensidade de 70 a $85 \%$ da FCmáx. Os exercícios anaeróbios eram realizados com halteres, duas a três vezes por semana, com duração de 30 a 45 minutos. Os protocolos resultaram em aumento de $15 \%$ e $3 \%$ do $\mathrm{VO}_{2}$ máx nos grupos de treinamento aeróbio e anaeróbio, respectivamente. No entanto, apesar da melhora nos testes de memória entre os períodos pré e pós treinamento, não houve diferenças entre os resultados dos testes de memória entre os grupos. 
No entanto, este achado não foi corroborado em um estudo mais recente com idosos saudáveis de ambos os gêneros, com 60 a 85 anos, não participantes de programas de treinamento físico (sedentários). Os voluntários foram distribuídos aleatoriamente em três grupos que foram submetidos a nove semanas de exercícios aeróbios, exercícios de força ou ausência de exercício. O programa de exercícios aeróbios consistia de caminhada na esteira em intensidade de $70 \%$ da FCR, três dias por semana. A duração dos exercícios iniciou com 20 minutos e foi aumentada em cinco minutos a cada sessão até que os voluntários pudessem caminhar por 50 minutos. O programa de exercício resistido consistia de 12 repetições de sete aparelhos diferentes que foram realizados em duas séries na primeira semana e três séries a partir da segunda semana. A carga, que inicialmente representava $60 \%$ de $1 \mathrm{RM}$, foi incrementada em $5 \%$ a cada duas semanas até que fossem atingidos $80 \%$ de 1RM. Ambos os grupos de exercício melhoraram seu desempenho cognitivo, porém, os melhores resultados foram vistos no grupo que realizou exercícios resistidos (Özkaya et al., 2005).

Componentes para o planejamento de um programa de treinamento: intensidade, freqüência e duração dos exercícios

De forma geral algumas considerações são necessárias quanto à intenção de planejar e prescrever atividades físicas, seja às pessoas saudáveis ou em casos especiais. As diferentes combinações entre estes exercícios implicarão em diferentes resultados. A freqüência do exercício refere-se ao número de vezes que o indivíduo se exercita por semana. A duração refere-se ao tempo despendido na execução de um exercício específico ou de uma sessão de exercícios. A intensidade, por sua vez, implica no componente qualitativo do treinamento, que está envolvido com a quantidade de trabalho realizado por unidade de tempo, assim, quanto mais trabalho em uma unidade de tempo fechada, maior será a intensidade (Dantas, 2003).

Neste sentido, alguns estudos objetivaram identificar quais destes parâmetros influenciam a cognição. Um deles comparou as respostas sobre ATF com testes cognitivos de 1.972 sujeitos com idades entre 45 e 70 anos. Com base nas respostas dadas nos questionários, era possível presumir a intensidade e o tempo total com que os voluntários realizavam suas ATF semanalmente. Os resultados demonstraram que os melhores desempenhos cognitivos estavam relacionados significantemente à intensidade e não à duração da ATF (Angevaren et al., 2007). A mesma conclusão havia sido encontrada anteriormente quando adultos jovens pedalaram a $80 \%$ da FCmáx durante sete, 20 e 40 minutos em dias diferentes. Os pesquisadores encontraram melhoras cognitivas em relação ao período pré exercício, independente do tempo que os participantes pedalaram, fato que não ocorreu quando os sujeitos pedalaram por 23, 57 e 110 minutos a 50\% da FCmáx (Higashiura et al., 2006).

Porém, uma associação entre a freqüência do treino e melhoras cognitivas foi encontrada em 71 sujeitos com idades entre 18 e 70 anos que completaram 10 semanas de treinamento aeróbio. Os voluntários praticaram exercícios de três a quatro ou de cinco a seis dias por semana a uma intensidade de $70-85 \%$ da FCmáx durante 30 a 45 minutos. Durante o mesmo período, 20 sujeitos que reportaram realizar exercícios menos de duas vezes por semana serviu como grupo controle. Ao final do estudo aqueles que praticavam de três a quatro e de cinco a seis vezes por semana demonstraram significante melhoras cognitivas, incluindo da memória. No entanto, as melhoras de maior magnitude foram observadas naqueles que se exercitaram mais de cinco vezes por semana (Masley, Roetzheim \& Gualtieri, 2009).

Em outro estudo, foram analisados os efeitos do exercício realizado em dias separados em intensidade leve, moderada e alta. Sujeitos com idades entre 22 e 30 anos foram avaliados através de eletroencefalograma durante testes cognitivos em níveis de dificuldades diferentes, realizados antes e imediatamente depois dos exercícios. Os resultados demonstraram que após os exercícios realizados em intensidade leve e moderada, a atividade eletroencefalográfica teve um aumento de sua amplitude em relação ao período pré exercício, fato que não ocorreu após o exercício em intensidade alta, indicando que deve haver um ponto na intensidade de execução do exercício em que a resposta cognitiva deva alcançar seu ápice e a partir daí, iniciar um declínio (Kamijo et al., 2009). Fato semelhante aconteceu com atletas recreacionais (não competitivos) submetidos a um protocolo de exercício em cargas crescentes até atingir o $\mathrm{VO}_{2}$ máx. Após o exercício, houve uma queda no desempenho cognitivo, sobretudo da memória quando comparados com os resultados pré exercício ou com o grupo controle (Covassin, Weiss, Powell \& Womack, 2007).

\section{Discussão e conclusão}

Existem fortes evidencias de que a ATF exerce efeitos benéficos sobre a memória. Seja em idosos ou adultos, os resultados são favoráveis à afirmação de que um estilo de vida que inclui ATF deve ser adotado para a prevenção dos declínios cognitivos vistos no processo de envelhecimento. Tal afirmação corrobora os resultados da revisão de Jedrziewski, Lee e Trojanowski (2007), que vai mais além, incluindo que a caminhada parece ser uma atividade aeróbia efetiva para uma cognição saudável. Na mesma direção, Hillman, Erickson e Kramer (2008) apontam que a ATF é o grande segredo para o bem estar cognitivo de todas as faixas etárias. Peters (2006) revisou o que se deve fazer para um envelhecimento biológico 
saudável e levantou que dentre a alimentação adequada, baixo ou moderado consumo de bebida alcoólica, abstinência de fumo e a ATF, a última ocupa um lugar de destaque, mostrando-se capaz de amenizar o declínio celular no cérebro.

Por sua vez, programas de exercícios aeróbios têm demonstrado algumas divergências quanto a seu benefício sobre a memória de idosos. Enquanto em alguns estudos (Kara et al., 2005; Peters, 2006), três meses de um protocolo de treinamento foram suficientes para melhorar a cognição em outro estudo, umprotocolo similar de seis meses não demonstrou evolução positiva (Madden et al., 1989). Apesar disso, em sua revisão, Angevaren, Aufdemkampe, Verhaar, Aleman e Vanhees (2008), encontraram evidências de que a melhora cardiovascular se relaciona às melhoras cognitivas eenfatizando que as diferenças entre os testes utilizados para acessar os domínios cognitivos nos estudos anteriores podem responder pelos resultados contraditórios encontrados.

As mesmas afirmações têm sido feitas por autores que avaliam os resultados agudos do exercício aeróbio sobre a cognição (Kamijo et al., 2007; Pontifex et al., 2009), justificando que os resultados dependem da intensidade do exercício e exigência cognitiva da tarefa. Além disso, Higashiura et al. (2006) levantam a hipótese da existência de uma curva em "U" invertida no desempenho da memória em relação à intensidade do exercício realizado, fato que, se confirmado, mudará o rumo dos estudos em exercícios físicos, memória e indivíduos idosos. No entanto, é plausível (e seguro) afirmar que os exercícios aeróbios realizados em intensidades moderadas apresentam resultados positivos sobre a memória em qualquer faixa etária (Netz et al., 2007; Kamijo et al., 2009).

Trabalhos que acessaram os efeitos dos programas de treinamento com exercícios resistidos sobre a memória são escassos e apenas o rigor metodológico do trabalho conduzido por Cassilhas et al. (2007), contribuiu para afirmar o benefício do treinamento com exercícios resistidos sobre a memória. Apesar das diversidades metodológicas, em sua revisão, LiuAmbrose e Donaldson (2009), concluem que existem evidências de que programas de exercícios resistidos atuam de forma positiva sobre a memória de idosos.

Quanto às respostas agudas do exercício resistido sobre a memória, apenas dois trabalhos foram encontrados. Assim, apenas Chang e Etnier (2009), demonstraram que uma sessão de exercícios resistidos, com intensidade de $75 \%$ de $1 \mathrm{RM}$ é suficiente para promover melhoras na memória e alguns outros domínios cognitivos. Há, porém, a necessidade de chamar a atenção ao fato de não terem sido observadas melhoras em todos os domínios cognitivos, despertando, a hipótese de que pode haver uma especificidade de resposta para alguns deles. É importante para o momento, que seja atentado a tal evento, uma vez que os achados de Chang e Etnier
(2009) confrontam-se com os de Pontifex et al (2009), Angevaren et al. (2008) e Kamina et al. (2007).

A comparação entre programas de exercícios resistidos e exercícios aeróbios foi feita por Özkaya et al. (2005) e Blumenthal e Madden (1988). Apesar de ambos os estudos demonstrarem que tanto o exercício aeróbio quanto o resistido resultam em benefícios na memória dos voluntários, o primeiro encontrou superioridade no programa de exercícios resistidos sobre os aeróbios e o segundo, não encontrou diferença alguma entre os exercícios aeróbios e os anaeróbios realizados com halteres. Portanto, apesar destas comparações deixarem poucas e vagas evidências, pesam em favor dos exercícios resistidos para o benefício da memória de idosos.

Por sua vez, apenas um trabalho, realizado com jovens, comparou os efeitos agudos promovidos pelo exercício resistido e aeróbio sobre a memória. Os resultados demonstraram que apenas os exercícios aeróbios melhoraram a memória. Apesar da impossibilidade de evidenciar a superioridade de um tipo de exercício sobre o outro quanto ao benefício à memória, o estudo suporta a hipótese de que a intensidade do exercício resulta em funcionamento cognitivo diferente em função da complexidade da tarefa exigida (Pontifex et al., 2009), portanto, contrariando a hipótese de que pode haver respostas diferentes segundo a complexidade dos testes em relação à intensidade do exercício (Kamijo et al., 2007; Higashiura et al., 2006). Fato comum é que ambas reforçam a crença na existência de uma interveniência não controlada que levanta a necessidade de revisar na literatura os tipos de testes usados para acessar os diferentes domínios cognitivos e comparar suas respostas pareadas pela intensidade. Contudo, este não era o objetivo do trabalho atual.

Quanto aos componentes para planejamento e prescrição adequada de um programa de treinamento/ tratamento com exercícios para beneficiar a memória, nota-se que ainda não há elementos suficientes para fazer recomendações. Os trabalhos são escassos e ainda não há como comparar os resultados para inferir duração, freqüência ou intensidade.

O treinamento aeróbio proposto por Masley et al. (2009), concluiu que a freqüência de cinco a seis vezes por semana traz o melhor benefício quando combinado a uma intensidade de 70-85\% da FCmáx e a duração 30-45 minutos, porém, os resultados ainda são únicos e podem até ser vistos como controversos, se levarmos em conta que Angevaren et al (2007) calculou o tempo semanal despendido na realização da ATF e quando comparado com a intensidade, concluiu que a manipulação deste segundo traz mais benefícios à cognição do que o tempo semanal gasto na ATF .

De outra forma, é proposto a existência de um ponto ideal da intensidade para as melhores respostas agudas da memória ao exercício aeróbio. Tal ponto 
parece surgir em uma intensidade entre moderado e alto (Kamijo et al., 2007; Covassin et al., 2007).

Há de se destacar, no entanto, que até o momento não existem trabalhos com comparações entre esses componentes e a resposta cognitiva aos exercícios resistidos.

Por fim, conclui-se que existem fortes evidências de que ATF e exercícios são benéficos à memória de idosos. Entretanto, são escassas as publicações que procuraram encontrar componentes específicos para a melhora da memória sem recorrer à melhora do desempenho físico. Porém parece haver uma tendência nos estudos ao empenho de encontrar parâmetros ideais para o treinamento ou tratamento objetivando melhorias à memória, mas até o momento, ainda não é possível estabelecer diretrizes com esse fim.

\section{Referências bibliograficas}

Angevaren, M., Aufdemkampe, G., Verhaar, H.J., Aleman, A., \& Vanhees, L. (2008). Physical activity and enhanced fitness to improve cognitive function in older people without known cognitive impairment. Cochrane Database Systematic Review, 16 (3), CD005381.

Angevaren, M., Vanhees, L., Wendel-Vos, W., Verhaar, H. J., Aufdemkampe, G., Aleman, A., \& Verschuren, W. M. (2007). Intensity, but not duration, of physical activities is related to cognitive function. European Journal of Cardiovascular Prevention \& Rehabilitation, 14 (6), 825-830.

Banich MT. Cognitive neuroscience and neuropsychology. 2nd. Ed. Boston: Houghton Mifflin, 2004.

Barnes, D, E., Yaffe, K., Satariano, W. A., \& Tager, I. B. (2003). A longitudinal study of cardiorespiratory fitness and cognitive function in healthy older adults. Journal of the American Geriatrics Society, 51 (4), 459-465.

Blumenthal, J. A., \& Madden, D. J. (1988). Effects of aerobic exercise training, age, and physical fitness on memory-search performance. Psychology and Aging, 3 (3), 280-285.

Cassilhas, R. C., Viana, V. A., Grassmann, V., Santos, R. T., Santos, R. F., Tufik, S., Mello, M. T. (2007). The impact of resistance exercise on the cognitive function of the elderly. Medicine and Science in Sports and Exercise, 39 (8), 1401-1407.

Chang, Y.K., \& Etnier, J. L. (2009). Effects of an acute bout of localized resistance exercise on cognitive performance in middleaged adults: A randomized controlled trial study. Psychology of Sport \& Exercise, 10 (1), 19-24.

Covassin, T., Weiss, L., Powell, J., \& Womack, C. (2007). Effects of a maximal exercise test on neurocognitive function. British Journal of Sports Medicine, 41 (6), 370-374.

Craik F.I.M, Winocur G., Palmer H., Binns M.A., Edwards M., Bridges K., Glazer P., Chavannes R., Stuss D. Cognitive rehabilitation in the elderly: effects on memory. JINS 2007;13:13242.

Dantas, E. H. M. (2003). A prática da educação física, (5ª ed.). Rio de Janeiro: Shape.

Fabre, C., Chamari, K., Mucci, P., Massé-Biron, J., \& Préfaut C. (2002). Improvement of cognitive function by mental and/ or individualized aerobic training in healthy elderly subjects. International Journal of Sports Medicine, 23 (6), 415-421.

Ferris, L. T., Willians, J. S., \& Shen, C. (2007). The Effect of Acute Exercise on Serum Brain-Derived Neurotrophic Factor Levels and Cognitive Function. Medicine and Science in Sports and Exercise, 39 (4), 728-734.

Gallucci, M., Antuono, P., Ongaro, F., Forloni, P. L., Albani, D., Amici, G. P., \& Regini, C. (2009). Physical activity, socialization and reading in the elderly over the age of seventy: what is the relation with cognitive decline? Evidence from "The Treviso Longeva (TRELONG) study". Archives of Gerontology and Geriatrics, 48 (3), 284-286.

Gleitman, H. (2002). Psicologia, (5 ${ }^{\mathrm{a}}$ ed.). Lisboa: Fundação Calouste Gulbenkian.

Guzowski, J. F., Lyford, G. L., Stevenson, G. D., Houston, F. P., McGaugh, J. L., Worley, P. F., \& Barnes, C. A. (2000). Inhibition of Activity-Dependent Arc Protein Expression in the Rat Hippocampus Impairs the Maintenance of Long-Term Potentiation and the Consolidation of Long-Term Memory. Journal of Neuroscience, 20 (11), 3993-4001.

Helene, A. F., \& Xavier, G. F. (2003). A construção da atenção a partir da memória. Revista Brasileira de Psiquiatria, 25 (suppl 2), 12-20.

Higashiura, T., Nishihira, T., Kamijo, K., Hatta, A., Lkim, S. R., Hayashi, K., Kaneda, T., \& Kuroiwa, K. (2006). The interactive effects of exercise intensity and duration on cognitive processing in the central nervous system. Advances in Exercise and Sports Physiology, 12 (1), 15-21.

Hillman, C. H., Erickson, K. I., \& Kramer, A. F. (2008). Be smart, exercise your heart: exercise effects on brain and cognition. Nature Reviews Neuroscience, 9 (1), 58-65.

Izquierdo, I. A., \& Medina, J. H. (1993). Role of amygdala, hippocampus and enthorinal cortex and memory consolidation and expression. Brazilian Journal of Medical and Biological Research, 26, 573-589.

Jedrziewski, M. K., Lee, V. M., \& Trojanowski, J. Q. (2007). Physical Activity and Cognitive Health. Alzheimer's \& Dementia, 3 (2), 98-108.

Johnson, W., Deary, I. J., McGue, M., \& Christensen, K. (2009). Genetic and environmental links between cognitive and physical functions in old age. The Journals of Gerontology Series B: Psychological Sciences and Social Sciences, 64 (1), 65-72.

Kamijo, K., Hayashi, Y., Sakai, T., Yahiro, T., Tanaka, K., \& Nishihira, Y. (2009). Acute effects of aerobic exercise on cognitive function in older adults. The Journals of Gerontology Series B: Psychological Sciences and Social Sciences, 64 (3), 356-363.

Kamijo, K., Nishihira, Y., Higashiura, T., \& Kuroiwa, K. (2007). The interactive effect of exercise intensity and task difficulty on human cognitive processing. International Journal of Psychophysiology, 65 (2), 114-121.

Kara, B., Pinar, L., Uğur, F., \& Oğuz, M. (2005). Correlations between aerobic capacity, pulmonary and cognitive functioning in the older women. International Journal of Sports Medicine, 26 (3), 220-224.

Kimura, N., Kazui, H., Kubo, Y., Yoshida, T., Ishida, Y., Miyoshi, N., Ogino, A., Doronbekov, T. K., Tokunaga, H., Ikejiri, Y., \& Takeda, M. (2009). Memory and physical mobility in physically and cognitively-independent elderly people. Geriatrics \& Gerontology International, 7 (3), 258-265.

Lambourne, K. (2006). The relationship between working memory capacity and physical activity rates in young adults. Journal of the American Geriatrics Society, 5, 149-153.

Larson, E. B., Wang, L., Bowen, J. D., McCormick, W. C., Teri, L., Crane, P., \& Kukull, W. (2006). Exercise is associated with reduced risk for incident dementia among persons 65 years of age and older. Annals of Internal Medicine, 144 (2), 73-81.

Liu-Ambrose, T., \& Donaldson, M. (2009). Exercise and cognition in older adults: is there a role for resistance training programmes. British Journal of Sports Medicine, 43 (1), 25-37.

Madden, D. J., Allen, P. A., Blumenthal, J. A., \& Emery, C. F. (1989). Improving aerobic capacity in healthy older adults does not necessarily lead to improved cognitive performance. Psychology and Aging, 4 (3), 307-320.

Masley, S., Roetzheim, R., \& Gualtieri, T. (2009). Aerobic exercise enhances cognitive flexibility. Journal of Clinical Psychology in Medical Settings, 16 (2), 186-193.

Mazzeo, R. S. (1991). Catecholamine responses to acute and chronic exercise. Medicine and Science in Sports and Exercise, 23 (7), 839-845.

Moscovitch M. Amnesia. In: Smesler NB \& Baltes OB. Eds The international encyclopedia of social and behavioral sciences (Vols. 1-26). Oxford: Pergamos/Elsevier Sciences, 2004

Netz, Y., Tomer, R., Axelrad, S., Argov, E., \& Inbar, O. 
(2007) The effect of a single aerobic training session on cognitive flexibility in late middle-aged adults. International Journal of Sports Medicine, 28 (1), 82-87.

Newson, R. S., \& Kemps, E. B. (2006). The influence of physical and cognitive activities on simple and complex cognitive tasks in older adults. Experimental aging research, 32 (3), 341362 .

Nieto, M. L., Albert, S.M., Morrow, L. A., \& Saxton, J. (2008). Cognitive status and physical function in older african Americans. Journal of the American Geriatrics Society, 56 (11), 2014-2019.

Özkaya, G. Y., Aydın, H., Toraman, F. N., Kızılay, F., Özdemir, O., \& Cetinkaya, V. (2005). Effect of strength and endurance training on cognition in older people. Journal of Sports Science and Medicine, 4, 300-313.

Perrig-Chiello, P., Perrig, W. J., Ehrsam, R., Staehelin, H. B., \& Krings F. (1998). The effects of resistance training on well-being and memory in elderly volunteers. Age and Ageing, 27 (4), 469475.

Peters, R. Ageing and the brain. (2006). Postgraduate Medical Journal, 82, 84-88.

Pontifex, M. B., Hillman, C. H., Fernhall, B., Thompson, K. M., \& Valentini, T. A. (2009). The effect of acute aerobic and resistance exercise on working memory. Medicine and Science in Sports and Exercise, 41 (4), 927-934.

Richards. M., Hardy, R., \& Wadsworth, M. E. (2003). Does active leisure protect cognition? Evidence from a national birth cohort. Social Science \& Medicine, 56 (4): 785-792.

Rosat, R. M., Chaves, M. L., Ribeiro, J. P., \& Izquierdo, I. A. (1990). The use of a new non-verbal test in the evaluation of recent memory. Brazilian Journal of Medical and Biological Research, 23 (9):805-809.

Sutoo, D., \& Akiyama. K. (2003). Regulation of brain function by exercise. Neurobiology of Disease, 13, 1-14.

Van Uffelen, J. G., Chin A Paw, M. J. M., Hopman-Rock, M. \& Van Mechelen, W. (2008). The Effects of Exercise on Cognition in Older Adults With and Without Cognitive Decline: A Systematic Review. Clinical Journal of Sport Medicine, 18 (6), 486-500.

Verghese, J., Lipton, R. B., Katz, M. J., Hall, C. B., Derby, C. A., Kuslansky, G., Ambrose, A. F., Sliwinski, M., \& Buschke, H. (2003) Leisure activities and the risk of dementia in the elderly. New England Journal of Medicine, 348 (25), 2508-2516.

Weuve, J., Kang, J. H., Manson, J. E., Breteler, M. M., Ware, J. H., \& Grodstein, F. (2004). Physical activity, including walking, and cognitive function in older women. Journal of the American Medical Association, 292 (12), 1454-1461.

Wiles, N. J., Haase, A. M., Gallacher, J., Lawlor, D. A., \& Lewis, G. (2007). Physical activity and common mental disorder: results from the Caerphilly study. American Journal of Epidemiology, 165 (8), 946-954.

Recebido: $17 / 03 / 2010$

Última Revisão: $31 / 05 / 2010$ Aceite Final: 16/06/2010

Sobre os autores

Helder Chiari - Centro de Estudos em Psicobiologia e Exercício - CEPE, Universidade Federal de São Paulo, UNIFESP

Marco Túlio de Mello - Centro de Estudos em Psicobiologia e Exercício - CEPE, Departamento de Psicobiologia, Universidade Federal de São Paulo, UNIFESP

Patricia Rezeak - Centro de Estudos em Psicobiologia e Exercício - CEPE, Universidade Federal de São Paulo, UNIFESP

Hanna Karen M. Antunes - Centro de Estudos em Psicobiologia e Exercício - CEPE, Departamento de Biociências, Universidade Federal de São Paulo, UNIFESP

Agradecimentos

Os autores agradecem o apoio técnico e financeiro da AFIP, CEPE, CEMSA, CEPIDSONO/FAPESP (processo número: 1998/14303-3) CNPq, INSTITUTO DO SONO e a FADA/UNIFESP. 